\begin{tabular}{|c|l|}
\hline Title & Number created by the interaction between consciousness and memory : A mathematical basis for preafference \\
\hline Author(s) & Tsuda, Ichiro \\
\hline Citation & $\begin{array}{l}\text { Integrative Psychological and Behavioral Science, 42(2), 153-156 } \\
\text { https://doi.org/40.1007/312124008-9062-y }\end{array}$ \\
\hline Issue Date & 2008-06 \\
\hline Doc URL & http://hdl.handle.net/2115/38534 \\
\hline Rights & The original publication is available at www.springerlink.com \\
\hline Type & article (author version) \\
\hline File Information & tsuda.pdf \\
\hline
\end{tabular}

Instructions for use 


\section{Editorial Manager(tm) for Integrative Psychological and Behavioral Science Manuscript Draft}

Manuscript Number:

Title: Number created by the interaction between consciousness and memory: A mathematical basis for preafference

Article Type: Original theoretical contribution

Keywords: number; chaotic dynamical systems; Markov partitions; intention; memory; time with a finite width.

Corresponding Author: Ichiro Tsuda,

Corresponding Author's Institution: Hokkaido University

First Author: Ichiro Tsuda

Order of Authors: Ichiro Tsuda 


\title{
Number created by the interaction between consciousness and memory: A mathematical basis for preafference
}

\author{
Ichiro Tsuda \\ Research Institute for Electronic Science, \\ Hokkaido University, \\ Sapporo, 060-0812, Japan
}

\begin{abstract}
I would like to deal with a process that numbers are generated by the interaction between consciousness and memory, associated with time with a finite width. It is pointed out that this process can be related with the emergence of preafference. It is concluded that only humans can create numbers, whereas animals simply produce 'random' sequences of symbols because they lack intentionality to search for an appropriate internal observation window that makes intention and memory compatible.
\end{abstract}

Key words: number; chaotic dynamical systems; Markov partitions; intention; memory; time with a finite width

\author{
Non-Newtonian time \\ Walter Freeman clarified the difference between (Newtonian) time and causation and \\ investigated its neural mechanism. He stated that the perceptions of time and causation
}


have their origins in the same intentional cycle, and thereby both are inextricably linked but differ in what they measure: time measures motion and cause measures intent. This difference can be formulated by taking into account sensori-motor interactions that operate by preafference in an action-perception cycle (1). On the other hand, the study of time associated with 'being' is difficult (2) because this type of time is created at higher cognitive levels, in a way that is different from Newtonian time. The latter simply parameterizes the motion of objects at each point in state space (phase space, in dynamical systems terminology), i.e., in an instant, irrespective of whether it is defined on a circle, a line, or more complicated geometric object. In this commentary, I would like to emphasize the importance of the emergence of non-Newtonian time in a cognitive process that may exhibit kairos-like character, which Freeman describes in the introductory part of his paper. For the first time, I will try to formulate the creation of a natural number by means of an interaction between consciousness and memory that is associated with non-Newtonian time with an arbitrary nonzero width. Moreover, Freeman does not clarify the relationship between chaotic dynamics and the action-perception cycle. I will try to show that there is a role for chaos in the action-perception cycle from a viewpoint that is different from conventional ones. The theory in this commentary can be viewed as an extension of Freeman's framework, to 
represent the potential micro-structure of action-perception cycle, based on the intent-memory cycle.

\section{Generation of natural numbers}

Now, let us generate a natural number, assuming that one exists. We consider the following situation. Assume that $A$ is the only person who can do $X$. Here, $X$ is arbitrary, for example, 'climbing Mt. Everest'. We recognize that the number of members in this group equals 1 . Next, $B$ allows himself or herself to do $X$. Then, we say that $B$ has become a member of the group in which only $A$ can do $X$. If $B$ joins this group, then we recognize that the number of group members is 2 , although we cannot say that $B$ belongs to the group in which only one person can do $X$. In order to avoid this contradiction, we have to imagine a situation in which $B$ must belong outside this group, even though he or she is qualified to be a member of the group. Again, another contradiction appears. The former situation is realized to be associated with intent or consciousness, in the sense that the second person enters into a group for which only one person has been qualified. On the other hand, the latter situation is realized to be associated with memory in the sense that before $B$ left the group, the number of group 
members was 2, which should have been memorized. At this stage, the number 2 is not yet realized but still must be simply memorized. The stronger the intention, the stronger the memory becomes. This positive feedback finally establishes a situation in which $A$ and $B$ are the only two who can do $X$. Then the number 2 has been realized. At the next stage, we can imagine another person $C$ being able to do $X$. The same situation may occur again. The number 3 may be created by an interaction between intention and memory.

In such a way, all the finite natural numbers can be created. Furthermore, we can imagine the process continuing towards infinity. Although we cannot imagine infinity itself, we can form an image of the process involved in a step-by-step recurrent creation of natural numbers. At each stage of number creation, a finite duration of time inevitably follows, associated with positive feedback provided by interactions between intention and memory. This whole process may be a cognitive alternative to a process of perception that involves both preafference and interactions with the environment.

At the final stage, we might be able to create the number 0 from 1 . $A$ was the first person who could do $X$. 
By the same logic as above, it can be considered that $A$ has entered into a group that is empty, that is, a null set. We may associate such a null set with the number 0 . However, this final stage is not straightforward. It seems to be rather vague when compared with the creation of natural numbers because the recognition of a null set is not trivial, and identifying the null set with the number 0 will involve thinking that is more abstract.

\section{Generation of real numbers}

Second, I would like to construct real numbers by means of chaotic dynamical systems characterized by a generator. A typical generator is a Markov partition. This kind of partition divides a state space into nonoverlapping subpartitions where one-to-one correspondence of the function that locally represents the dynamical rule is assured, and subdivisions divide the state space into single points once an infinite number of subdivisions have taken place. In chaotic dynamical systems, a dynamical orbit starts from an initial condition. Nearby orbits separate from each other exponentially in time. How can we determine an initial position in state space using information about future orbits? Assume that the state space is divided into two parts as the first Markov partition, one of which is labeled by a symbol, say $L$, and the other by, say $R$. By this labeling, we 
can assign a symbol sequence to a dynamical orbit by associating either the symbol $L$ or $R$ with the appropriate partition that the orbit entered. In other words, for each dynamical orbit up to a certain finite (Newtonian) time, a partition of a finite width in the state space is associated with the creation of a corresponding symbol sequence with a finite length in the symbol space. Actually, the state space can be divided into Markov partitions, if they exist. Each partition of which represents a certain symbol sequence that correctly characterizes a chaotic orbit starting from a given initial condition. For any infinitely long symbol sequence, a point in state space is determined. This point maintains information about the initial condition of such an orbit in the sense that there exists a functional relation between such a point and an initial condition. In a special case, such a point is exactly the initial point of the orbit. Therefore, we can say that an infinitely long sequence that continues far into infinite future determines the initial point from which the chaotic orbit starts.

\author{
If we replace the symbols, $L$ and $R$, with the numbers, 0 and 1 , respectively, the symbol \\ sequence can be replaced by a number sequence that can be viewed as a binary \\ expansion of a certain real number. In such a way, we generate a real number that is \\ associated with each division point in an infinite Markov partition. In this way, all real
}


numbers can be created by means of a chaotic dynamical system using a Markov partition as its generator.

\section{Simple example}

Let us provide a simple example. We consider a chaotic dynamical system, $Z_{\mathrm{n}+1}=2 Z_{\mathrm{n}}$

$(\bmod 1)$, where $Z$ is a state variable and $n$ denotes a discrete time. This dynamical system is realized by taking only the decimal part of number in each iteration, starting from an arbitrary number between 0 and 1 . Here, the state space is a unit interval. We divide the unit interval into two subintervals, centered at 0.5 . We associate the number 0 with the left subinterval and 1 with the right one. If we start with $Z=0.1$, we obtain the dynamical orbit, $\{0.1,0.2,0.4,0.8,0.6,0.2,0.4,0.8, \ldots\}$. This orbit is an eventually periodic but unstable orbit, $\{0.2,0.4,0.8,0.6\}$. The assigned number for the partition is $0001100110011 \ldots . .$, , including a symbol corresponding to a transient orbit. If this is viewed as a binary expansion of some number between 0 and 1, it turns out that this number represents 0.1 , which is exactly the initial condition. This relation also holds for the chaotic orbits. Instead of assigning the number 0 or 1 to the orbit at each instant of time, we can divide the state space more finely by applying a higher Markov partition. 
The highest order of Markov partition can divide the space into an infinite number of points. Then, each point represents an infinite sequence consisting of 0 and 1 that defines a real number. In this way, an infinite Markov partition defines an initial condition, and such a partition represents an infinitely long orbit from the initial condition to the infinite future.

\section{Ill-posedness and preafference}

These considerations may provide a hypothesis regarding the biological significance of embedding chaotic dynamics into neural systems. Perhaps, when animals or humans act upon their environment, the internal chaotic dynamics embedded in neural networks will generate numbers. Usually, however, such numbers are simply 'random' because of the ill-posedness of any scheme for obtaining information about the initial state of a system using a sequence of symbols from its initial state to its final state. By active interaction with the environment via the action-perception cycle, a generator such as a Markov partition may be searched by changing its own chaotic dynamics. Once such a partition has been found, animals or humans can correctly obtain information about the initial state, and therefore also information about any current states. Thus, generating 
correct numbers may establish preafference. When considered together with Freeman's ideas on the difference between (Newtonian) time and cause, only humans can create numbers, whereas animals simply produce 'random' sequences of symbols because they lack intentionality to search for an appropriate partition that makes intention and memory compatible.

\section{References}

(1) Kay LM, Freeman WJ (1998) Bidirectional processing in the olfactory-limbic axis during olfactory behavior. Behavioral Neuroscience 112: 541-553.

(2) Diebner HH, Tsuda I (2005) Fundamental interfaciology: Indistinguishability and Time's arrow. The Proc. of the Third Conference on Information Science. MDPI. http://www.mdpi.org/fis2005/

\section{BIOGRAPHICAL SKETCH}

Ichiro Tsuda holds the position of Professor in the Research Institute of Electronic Science (RIES), and the Graduate School of Mathematics, Hokkaido University, as well as Visiting Professor at Department of Mechanical Engineering, Osaka University. He has published widely in the field of chaotic dynamical systems and the brain science. 
His research interest is mathematical modelling of the higher brain function including memory dynamics, thoughts, and inference processes, and also numerical studies of chaotic dynamical systems. He constructed a one-dimensional map for sufficiently explaining chaos and bifurcation structure in the BZ reaction, and found noise-induced order in such a model. He also constructed neural network models for dynamic associative memory and also for episodic memory, based on physiological data, where he found a new transitory dynamics, named chaotic itinerancy, and proposed its dynamical interpretation in terms of Milnor attractor. He is also an editor of scientific journals of Neural Networks, J. of Cognitive Neurodynamics and others, and also an advisory board of Chaos. 